\title{
GI-MS46-P06 | DEVELOPMENT OF MICROSPECTROPHOTOMETER FOR THE macromolecular crystallography beamline at the Photon Factory, Japan
}

Hikita, Masahide (Structural Biology Research Center, KEK, Tsukuba, JPN); Yamada, Yusuke (Structural Biology Research Center, KEK, Tsukuba, JPN); Matsugaki, Naohiro (Structural Biology Research Center, KEK, Tsukuba, JPN); Hiraki, Masahiko (Mechanical Engineering Center, KEK, Tsukuba, JPN); Senda, Toshiya (Structural Biology Research Center, KEK, Tsukuba, JPN)

In the functional analysis of proteins, it is essential to know the crystal structure at the atomic level and its electronic and chemical changes. Spectroscopy is the effective technique for detecting the structural states of proteins, even within protein crystals. Therefore, the spectroscopic methods such as Raman and UV-Visible absorption have been complementarily combined with X-ray diffraction studies to evaluate the protein status. In order to utilize the spectroscopic study at macromolecular crystallography beamline at the Photon Factory, the development of spectroscopic instrumentation for both offline and online has been started at beamline ARNW12A. For development of offline spectroscopic instrumentation, the laser booth has been built beside of control cabin of AR-NW12A. After the end of the construction of the laser booth, we have started to develop the offline UV-Visible absorption spectroscopic instrumentation and it has now been in general user operation. Herein, we describe the outline of the offline UV-Visible and Raman spectroscopic instrumentations. The continuing development of the online spectroscopic instrumentation is also outlined. In the future, macromolecular crystallographic beamline users will be able to not only determine the atomic structure of their samples but also to explore the electronic and vibrational characteristics of their sample, before, during and after data collection. 